\title{
DISTRIBUTION OF RISK FACTORS IN PATIENTS WITH ACUTE CORONARY SYNDROME - A HOSPITAL BASED STUDY
}

\author{
KC Man B*, Rajbhandari S*, Sharma D*, Malla R*, \\ Limbu Y R*, Rajbhandari R*, Regmi S R*, Maskey A*
}

\section{ABSTRACT}

A total of 230 patients admitted in coronary care unit of Shahid Gangalal National Heart Centre in between November 2001 and October 2002 were studied. 157 (68.2\%) were male, with mean age of 62.8 \pm 11.8 years. Majority of acute coronary syndrome (ACS) events were observed in males with advanced age ( $>65$ years). About 68\% patients with ACS were smoker, the most common modifiable risk factor followed by hypertension, dyslipidaemia and diabetes. A strong trend towards development of coronary artery disease (CAD) was found in subjects with positive family history of CAD. Multiple risk factors ( 2 or more) were present in vast majority of patients. Modifiable risk factors, with smoking at number one position are more common in acute coronary syndrome patients. Appropriate management of modifiable risk factors is advisable to reduce the incidence of coronary artery disease.

Key Words: Coronary Artery Disease, Risk Factors.

\section{INTRODUCTION}

Coronary artery disease (CAD) is emerging as a growing epidemic in whole of the South Asia region. South Asians are genetically most predisposed people to CAD. In this background of genetic predisposition to $\mathrm{CAD}$ and changes in life style, which increases the vulnerability to acquire this disease, hospital admissions due to CAD are increasing every year. $^{1,2}$ There are already well established risk factors of CAD of which some are modifiable, like smoking, and some are not like heredity, age and male sex. Presence of major risk factors like smoking, hypertension, diabetes and dyslipidaemia, alone or in combination, increase the predisposition to premature and severe CAD. In this article, an attempt is made to assess distribution of risk factors in patients with acute coronary syndrome (ACS) admitted in coronary care unit (CCU) of Shahid Gangalal National Heart Centre (SGNHC).

\section{SUBJECTS AND METHODS}

The study included retrospective analysis of 230 patients, out of which $157(68.2 \%)$ were male and rest were female. They were admitted in CCU of SGNHC from November 2001 to October 2002 with the diagnosis of ACS (i.e. Acute ST elevation MI, Non ST elevation MI and Unstable Angina). The mean age of the patients was $62.88 \pm 11.71$ years. Data were collected from inpatient hospital records of SGNHC and were analyzed as required. Risk factors identified were smoking, hypertension, diabetes mellitus, dyslipidaemia, obesity, family history of premature CAD, advanced age and male sex. Smoking was defined as continuous use of cigarette and / or any form of tobacco. Hypertension was considered with the BP of more than $140 / 90 \mathrm{mmHg}$ at more than two occasions, taken at different times or in patients previously

* Shahid Gangalal National Heart Centre, Bansbari, Kathmandu, Nepal.

Address for correspondence : Dr. Man Bahadur K.C.

Department of Cardiology

Shahid Gangalal National Heart Centre, Bansbari, Kathmandu

Email: manbdrkc@yahoo.com 
diagnosed as hypertensives. Diabetes mellitus was diagnosed on the basis of fasting and post-prandial blood glucose or from patient's previous medical records. Dyslipidaemia was considered when total cholesterol $>200 \mathrm{mg} / \mathrm{dl}$ and / or, LDL $>100 \mathrm{mg} / \mathrm{dl}$ and / or, HDL $<40 \mathrm{mg} / \mathrm{dl}$ and / or TG $>150$ $\mathrm{mg} / \mathrm{dl}$. Obesity was defined as BMI of $>30 \mathrm{Kg} / \mathrm{M}^{2}$. Family history of premature CAD was defined as history of CAD in the first degree relatives at an early age (male $<55$ years and females $<65$ years). Demographic data were collected and expressed as percentage, mean and standard deviation wherever applicable. Two sided ' $t$ ' test was used to estimate ' $p$ ' value, and 'p' value of less than 0.05 was considered to be statistically significant.

\section{RESULTS}

Highly significant $(\mathrm{P}<0.001)$ risk of developing $\mathrm{CAD}$ was observed in male sex and advanced age (male $>55$ and female $>65$ ). Smoking was observed as the commonest modifiable risk factor followed by hypertension, dyslipidaemia and diabetes mellitus (Table I). A strong trend was noted for premature development of CAD in presence of positive family history of $\mathrm{CAD}$, smoking and diabetes mellitus alone or in combination. Multiple risk factors ( 2 or more) were present in the vast majority of patients (Table II). There was an interesting correlation observed in between presence of multiple risk factors and higher chances of development of extensive myocardial infarction, hemodynamic derangement and premature CAD.

\section{DISCUSSIONS}

In our study advanced age and male sex were most common risk factors of CAD patients. But both are non - modifiable risk factors and cannot be changed. Among the other modifiable and partially modifiable risk factors, smoking was the most common $(68.7 \%)$, followed by hypertension $(47 \%)$, dyslipidaemia (37\%), diabetes mellitus (24.8\%) and obesity $(20 \%)$. In a study done in Kathmandu by Bharat Rawat et al, ${ }^{3}$ the distribution of risk factors in male sex was almost similar with our study.

Smoking is already established major and independent risk factor for CAD. Studies have suggested that smoking more than 20 cigarettes a day results in two to three fold increase in total CAD. ${ }^{4}$ These effects depend on dose, duration of smoking and starting age of smoking. Consumption of as few as 4 cigarettes per day increases the risk of CAD. Smoking acts synergistically with other risk factors of CAD like oral contraceptives, hypertension, diabetes mellitus and dyslipidaemia. In addition to $\mathrm{CAD}$, smoking directly relates to increased rates of sudden death, aortic aneurysm formation, peripheral vascular disease and stroke. ${ }^{5}$ Long term smoking enhances the oxidation of LDL-C and decreases HDL-C, impairs coronary vasodilatation, increases acute phase reactants like CRP, increases fibrinogen level, causes platelet aggregation and increase monocyte adhesion to endothelium. ${ }^{3}$ smoking cessation alone reduces the risk of a first attack by nearly $65 \%{ }^{6}$

Systemic arterial hypertension is also a major independent risk factor of CAD. The risk of development of CAD is parallel to level of diastolic blood pressure. In hypertensive subjects there is $27 \%$ increase in the risk of $\mathrm{CAD}$, for every $7 \mathrm{mmHg}$ increase in diastolic blood pressure and reduction of diastolic blood pressure by 5 to $6 \mathrm{mmHg}$ reduces the risk of CAD by $14 \%$ and risk of stroke by $40 \% .^{7}$

Table II : Single / Multiple Risk Factors in Patients with ACS 